\section{Is There Room for a New Diagnostic Subtype-The Schizo-obsessive Subtype?}

BY JUSEPH ZIHAR, MD

\section{INTRDDUCTIDN}

The concept of diagnosis, ie, collecting a group of symptoms under the umbrella of a disease or disorder, serves a number of important goals. It assists in treatment, might have some prognostic value, supports communication between physicians, and, finally, it could have implications regarding the pathological basis of a disorder. All four components would not be required to bring about a new diagnostic subpromoting communication and probably providing a clearer prognosis, might justify establishing a new diagnostic subtype. Especially if a new therapy, based on the new subtype, was suggested (which could, in some cases, actually alter the prognosis).

\section{SCHIZD-DBSESSIVE DISDRDER}

Is there enough data to suggest that we should begin to consider schizo-obsessive disorder as a new subtype of schizophrenia?

Two studies have looked at the prognosis of patients with OCD and schizophrenia. ${ }^{1.2}$ Neither is prospective. Both found the prognosis of these patients to be poorer than it would be for those with only one disorder. This is not entirely surprising, since in many cases comorbidity predicts type. Even simply grouping symptoms, thereby poor prognosis. However, if we examine the schizoaffective subtype, which usually has a better prognosis than schizophrenia, this is not always the case.

What about specific treatment? Although the data is limited (less than 100 patients with these disorders have been studied) there is some indication that this subset of patients may respond to a combination of antipsychotic and antiobsessive medications. ${ }^{3.4}$

One advantage of a new diagnostic subtype is that it could shape the way in which we process clinical observation. As far as the medical profession is concerned, the importance of establishing a new diagnostic subtype for a disorder lies in its ability to offer the clinician a better tool with which to determine prognosis, improve treatment, and achieve effective communication between clinicians.

The reliability of a diagnosis, ie. its reproducibility among different psychiatrists for the same patient at different points in time, is a crucial element for any diagnosis, let alone a new one. This has been a problem with individual subtypes which often change. In the case of the schizo-obsessive subtype, in which the combination of two well-diagnosed disorders is proposed,
"As far as the

medical profession

is concerned, the

importance of

establishing a new

diagnostic subtype for

a disorder lies in its

ability to offer the

clinician a better

tool with which to

determine prognosis,

improve treatment,

and achieve effective

communication

between clinicians."

Dr. Zohar is the international

Pathogenesis

\section{Communication}

editor of this journal.

He is also professor, Department of Psychiatry, Chaim Sheba Medical Center. Tel Aviv, Israel. 
the reliability that has been established in these disorders individually could serve as a solid basis for the new subtype.

One key element in a new classification is that it suggests another way to interpret the clinical observation of the data. For example, the distinction between stereotypical behavior

"Although very little

is known about

the pathological basis

of schizo-obsessive

patients, it appears

that they respond

with an initial

exacerbation of

obsessive-compulsive

symptoms when

given a new, atypical

antipsychotic such

as clozapine

and olanzapine." and compulsion is often unclear to the observer (although it is clear to the patient). By accepting the possibility of the schizo-obsessive subtype, the clinician might be encouraged to ask more specific questions about what would otherwise be interpreted as either a stereotypy egosyntonic behavior, dictated from the outside, or as bizarre, delusion-driven thoughts. Thus, after appropriate questioning, what might emerge is an egodystonic ritual or obsession that the patient is well aware originates in his or her own mind, and which he or she would like to minimize or avoid altogether, but cannot.

Pathology is hard to attain. It is the hope that a proper diagnosis would enable us to study less heterogenic pathognomonic groups and enhance the specificity of results obtained by sampling these groups. Although very little is known about the pathological basis of schizo-obsessive patients, it appears that they respond with an initial exacerbation of obsessive-compulsive symptoms when given a new, atypical antipsychotic such as clozapine ${ }^{5}$ and olanzapine. However, a combination of risperidone and antiobsessive medications has been reported to be a useful augmenting strategy in therapy resistant patients. ${ }^{6}$

Although we attempt to diagnose every disorder, even rare ones, the impact of a new diagnostic subtype has to do with the prevalence of the disorder under discussion. The prevalence of OCD among schizophrenic patients is between $10 \%$ and $40 \%,{ }^{2}$ which makes this subtype a frequent for the clinician, who therefore may be encountering the schizo-obsessive patient quite often.

\section{CDNCLUSIDN}

In the past five years there have been an increasing number of publications on different aspects of schizo-obsessive disorder. The findings so far suggest that this subtype involves poor prognosis, specific response to a combination of antipsychotics and SRIs, and might show a different response to some of the new antipsychotic medications. These findings, coupled with the high prevalence of this subtype among schizophrenic patients, makes this a timely issue. Moreover, proposing the schizo-obsessive subtype as a new subtype of schizophrenia exemplifies the interpretation of clinical observations from a new perspective. The findings also point to a different prognosis, suggest another therapeutic approach, and may reflect a different psychopathology.CNS

\section{REFERENCES}

1. Fenton WS. McGlashan TH. The prognostic significance of obsessive-compulsive symptoms in schizophrenia. Am J Psychiatry. 1986;143:437-441.

2. Berman I, Kalinowski A, Berman SM, et al. Obsessive and compulsive symptoms in chronic schizophrenia. Comprehen Psychiatry. 1995;36:6-10.

3. Zohar J, Kaplan Z, Benjamin J. Clomipramine treatment of obsessive compulsive symptomatology in schizophrenic patients. J Clin Psychiatry. 1993;54:385-388.

4. Berman I, Sapers BL, Chang HHJ, et al. Treatment of obsessive-compulsive symptoms in schizophrenic patients with clomipramine. J Clin Psychopharmacol. In press.

5. Baker RW, Chengappa KNR, Baird JW, et al. Emergence of obsessive compulsive symptoms during treatment with clozapine. J Clin Psychiatry, 1992:53:439-441.

6. McDougle CJ, Fleischmann RL, Epperson CN, et al. Risperidone addition in fluvoxamine-refractory obsessivecompulsive disorder: three cases. J Clin Psychiatry. 1995; 56:526-528. 